\title{
Contribution of Individual Judgments towards Inconsistency in Pairwise Comparisons
}

\author{
Sajid Siraj ${ }^{\mathrm{a}, \mathrm{b}, *}$, Ludmil Mikhailov ${ }^{\mathrm{c}}$, John A. Keane $^{\mathrm{d}}$ \\ ${ }^{a}$ Portsmouth Business School, Richmond Building, Portland Street, Portsmouth PO1 3DE, United Kingdom \\ ${ }^{b}$ Department of Electrical Engineering, COMSATS Institute of Information Technology, Wah Cantonment, Pakistan \\ ${ }^{c}$ Manchester Business School, Booth Street West, The University of Manchester, M15 6PB, United Kingdom \\ ${ }^{d}$ School of Computer Science, The University of Manchester, M13 9PL, United Kingdom
}

\begin{abstract}
Pairwise comparison (PC) is a well-established method to assist decision makers in estimating their preferences. In PCs, the acquired judgments are used to construct a PC matrix (PCM) that is used to check whether the inconsistency in judgments is acceptable or requires revision. The use of Consistency Ratio (CR) - a widely used measure for inconsistency - has been widely debated and the literature survey has identified a need for a more appropriate measure. Considering this need, a new measure, termed congruence, is proposed in this paper. The measure is shown to be useful in finding the contribution of individual judgments towards overall inconsistency of a PCM and, therefore, can be used to detect and correct cardinally inconsistent judgments. The proposed measure is applicable to incomplete sets of PC judgments without modification, unlike $\mathrm{CR}$ which requires a complete set of PC judgments. To address ordinal inconsistency, another measure termed dissonance, is proposed as a supplement to the congruence measure. The two measures appear useful in detecting both outliers and the phenomenon of consistency deadlock where all judgments equally contribute towards the overall inconsistency.
\end{abstract}

Keywords: Decision support systems; Multi-criteria decision making; Pairwise comparison; Intuitionistic preference modeling; Analytic hierarchy process.

\section{Introduction}

The method of pairwise comparison (PC), introduced by (Thurstone, 1927), has been used in several decision making methods to assess the relative importance of criteria and alternatives, see (Saaty, 2008; Siskos et al., 2005; Figueira et al., 2004; Greco et al., 2010) for examples. The main benefit of using this

*Sajid Siraj, Portsmouth Business School, Richmond Building, Portland Street, Portsmouth PO1 3DE, United Kingdom. Tel: +9251 9272614, Fax: +92514546850

Email address: sajidsiraj@comsats.edu.pk (Sajid Siraj) 
approach is to convert both objective and subjective judgments into relative weights of importance. This paper focuses on the method of pairwise comparisons that is often used to help DM in describing his/her preferences. A new technique is proposed here to measure the level of inconsistency in a set of pairwise judgments.

The pairwise judgments can be used to construct a matrix and a prioritization procedure can be applied to derive a corresponding priority vector. If the judgments are consistent then all prioritization methods give the same result. However, in the case of inconsistent judgments, different prioritization methods derive different priority vectors (Choo and Wedley, 2004). It is therefore important to measure consistency of judgments prior to the process of prioritization.

Although widely used, current measures for consistency have been criticized for their shortcomings (see details in Section 2.2). We contend that the redundancy in PC judgments can be used to measure the contribution of each judgment towards the overall inconsistency. Based on this, two new measures for consistency are proposed here and are shown to be useful in measuring overall inconsistency in PC judgments. Moreover, these measures can also be used to suggest changes in the judgment values in order to improve overall consistency.

The rest of the paper is structured as follows: Section 2 formulates the problem of prioritization and reviews the literature related to consistency in PC judgments; Section 3 collects an empirical evidence that existing measures are insufficient; the new measures are then proposed in Sections 4 and 5 respectively. Section 6 discusses the use of these measures for improving the overall consistency in PC judgments and then illustrated through examples in Section 7; finally, Section 8 concludes the paper.

\section{Background}

Consider a prioritization of $n$ elements $E_{1}, E_{2}, \ldots, E_{n}$. In the PC method, DMs assess the relative importance of any two elements $E_{i}$ and $E_{j}$ by providing a ratio judgment $a_{i j}$, specifying by how much $E_{i}$ is preferred to $E_{j}$. If the element $E_{i}$ is preferred to $E_{j}$ then $a_{i j}>1$, if the elements are equally preferred then $a_{i j}=1$ and if $E_{j}$ is preferred to $E_{i}$ then $a_{i j}<1$. These judgments can be used to construct a PC matrix $(\mathrm{PCM}), A=\left[a_{i j}\right]$, of the order $n \times n$. This includes all self-comparison $\left(a_{i i}=1\right)$ and reciprocal judgments $\left(a_{j i}=\frac{1}{a_{i j}}\right)$. The prioritization problem is to determine a priority vector $w=\left(w_{1}, w_{2}, \ldots, w_{n}\right)^{T}$ from the given set of judgments, $a_{i j}$. There exist many prioritization methods that can be applied to derive a priority vector from a set of PC judgments (Choo and Wedley, 2004).

The relationships among elements can also be depicted by means of a directed graph (digraph), $G=$ 
$(E, J)$, where $E$ is the set of nodes representing $n$ elements $E_{1}, E_{2}, \ldots, E_{n}$, and $J$ represents the set of all ratio judgments $\left\{a_{i j}\right\}$ as weighted edges (Ali et al., 1986; Harker and Vargas, 1987). When a complete set of judgments is provided, the digraph $G$ becomes fully connected.

\subsection{Judgment Scale}

The cardinal information, $a_{i j}$, is provided with respect to some predetermined preference scale. In the case of tangible criteria, this can be derived from the directly measured information as, for example, weights (in kgs) or price (in euros). In the case of intangibles, Saaty (1977) proposed the use of a set of verbal judgments that correspond to the ratio-scale of 1 to 9 . Pairwise judgments can be provided using an additive-scale, however, the use of ratio-scale is considered to be more appropriate for measuring the relative intensities (Harker and Vargas, 1987; Krantz, 1972; Stevens, 1961). Despite its criticism and shortcomings (Dyer, 1990; Belton and Gear, 1983), the 1 to 9 scale has been widely used in various applications (Vaidya and Agarwal, 2006; Vargas, 1990).

Harker and Vargas (1987) compared Saaty's scale with other proposed scales and considered it to be more appropriate than others. In their comparison, the proposed scales included two linear scales, 1-5 and 1-15, and two non-linear ones, $x^{2}$ and $\sqrt{x}$. Their claim was, however, supported by only one example, leaving the argument largely unresolved. Lootsma (1993) preferred the use of a geometric scale over the linear one, while Salo and Hamalainen (1997) proposed a balanced scale claiming that the weights generated by their proposed scales are more evenly dispersed as compared to Saaty's scale. Finan and Hurley (1999) proposed to re-calibrate the verbal scale, transforming it into a geometric scale and claimed to achieve better performance compared with linear scales. Saaty (1994) also proposed a finer-grained version of the 1 to 9 scale, where he suggested the use of 1.1 to 1.9 for comparing stimuli with close resemblance. Ishizaka et al. (2011) summarized a comprehensive list of transformations applied to Saaty's 1-9 scale with a conclusion that there exists no single scale appropriate for all situations.

A complete set of judgments in the PC method creates an opportunity to have inconsistent information, primarily due to the redundancy inherent in its structure. One may argue to try to avoid redundancies present in data, however, redundancy may prove valuable in validating information acquired from imperfect human minds.

\subsection{Inconsistencies in Judgments}

There are several causes of inconsistency including psychological reasons, clerical errors and an insufficient model structure (Sugden, 1985). The psychological reasons further break down into factors such as 
incomplete information, uncertainty and/or lack of concentration during the judgment process.

The judgment scale of 1 to 9 (Saaty, 1980) is an appropriate example of an insufficient model structure. Consider that if $\mathrm{A}$ is 5 times better than $\mathrm{B}$ and $\mathrm{B}$ is 3 times better than $\mathrm{C}$, then the consistent value of 15 for comparing $\mathrm{A}$ to $\mathrm{C}$ is unavailable when using the 1 to 9 scale. Also, replacing these values with verbal statements will not change the underlying model/scale, and therefore, using the linear scale has this limitation in its structure.

There are situations where inconsistencies are unavoidable e.g. a tournament ranking problem including results where A beats $\mathrm{B}$ and $\mathrm{B}$ beats $\mathrm{C}$, but then $\mathrm{A}$ loses to $\mathrm{C}$ (intransitivity). In this situation, ranking is required without amending any data, as teams may be unable to play again. Sugden (1985) suggested such inconsistencies be considered as rational.

Another possible cause of inconsistency is incorrect data entry e.g. entering a reciprocal value into a PCM. This happens when a ratio for $E_{i}$ to $E_{j}$ is mistakenly provided at a transpose location in a PCM i.e. $a_{j i}$ instead of $a_{i j}$. Lipovetsky and Conklin (2002) termed such errors Unusual and False Observations (UFO).

Consistency in PCs is generally of two types i.e. cardinal consistency (CC) and ordinal consistency (OC). The judgments of DMs are cardinally consistent, if $a_{i j}=\frac{1}{a_{j i}}$ and $a_{i j}=a_{i k} a_{k j}$ for all $i, j$ and $k$. OC states that if $E_{i}$ is preferred to $E_{j}$ and $E_{j}$ is preferred to $E_{k}$, then $E_{i}$ should be preferred to $E_{k}$ i.e. If $E_{i} \rightarrow E_{j} \rightarrow E_{k}$ then $E_{i} \rightarrow E_{k}$.

The preference judgments are ordinally inconsistent (or intransitive) if $E_{k} \rightarrow E_{i}$ when $E_{i} \rightarrow E_{j} \rightarrow E_{k}$. Therefore, ordinal inconsistency can be defined as $E_{i} \rightarrow E_{j} \rightarrow E_{k} \rightarrow E_{i}$, which represents a circular triad of preferences (Kendall and Smith, 1940).

When two comparison elements $E_{i}$ and $E_{j}$ are equally preferred, $E_{i} \sim E_{j}$ (preference equivalence or a tie), then $\mathrm{OC}$ requires one of the following three conditions to be satisfied: $E_{i} \rightarrow E_{k}$ and $E_{j} \rightarrow E_{k}$, or $E_{i} \leftarrow E_{k}$ and $E_{j} \leftarrow E_{k}$, or $E_{i} \sim E_{k}$ and $E_{j} \sim E_{k}$. For example, if $a_{i j}=1$ and $a_{k i}>1$, then OC demands that $a_{k j}>1$. This implies that a judgment $a_{k j}<1$ will introduce ordinal inconsistency. The ties present in PCMs can be shown as undirected edges between corresponding elements (Jensen and Hicks, 1993).

\subsubsection{Eigenvalue-based Measures}

Using the fact that for a consistent positive reciprocal matrix, the largest Eigenvalue $\lambda_{\max }$ is equal to $n$, Saaty defined a measure of consistency, termed a Consistency Index (CI):-

$$
C I=\frac{\lambda_{\max }-n}{n-1}
$$




\begin{tabular}{lcc}
\hline$n$ & (Saaty, 1980) & (Aguaron and Moreno-Jimenez, 2003) \\
\hline $\mathbf{3}$ & 0.58 & 0.5245 \\
$\mathbf{4}$ & 0.90 & 0.8815 \\
$\mathbf{5}$ & 1.12 & 1.1086 \\
$\mathbf{6}$ & 1.24 & 1.2479 \\
$\mathbf{7}$ & 1.32 & 1.3417 \\
$\mathbf{8}$ & 1.41 & 1.4056 \\
$\mathbf{9}$ & 1.45 & 1.4499 \\
\hline
\end{tabular}

Table 1: Random Consistency Indices

where perfect consistency implies that $C I=0$ and $C I>0$ for inconsistent matrices.

To define a unique consistency measure which does not depend on the dimension of the PCM, Saaty (1980) further introduced the Consistency Ratio $(C R)$ criterion. This is the ratio between the Consistency Index and a Random Consistency Index $(R I)$ :-

$$
C R=\frac{C I}{R I}
$$

where $R I$ represents the average $C I$ of a randomly generated PCM of the same dimension, $n$. The values of $R I$ are statistically calculated from thousands (or millions) of randomly generated PCMs.

Table 1 shows the two sets of CR values given in (Saaty, 1980) and (Aguaron and Moreno-Jimenez, 2003). Aguaron and Moreno-Jimenez (2003) calculated the values of CR by increasing the number of iterations for their experiments and, therefore, claiming higher precision.

Saaty (1980) claimed that if the value of CR is smaller than or equal to 0.1 , the estimated priority vector $w$ can adequately approximate the unknown preference vector $r$. Therefore, the PCM is considered to be acceptable when the threshold of $C R \leqslant 0.1$ is met. However, if $C R>0.1$, the estimated priorities could be erroneous and, therefore, DMs should be asked to improve the consistency by revising their subjective judgments. This threshold is used in various applications to accept or reject a PCM (Vaidya and Agarwal, 2006).

Although widely used, CR has been much debated for its two major drawbacks i.e. sensitivity to scale (Murphy, 1993; Stein and Mizzi, 2007) and the threshold value of $C R \leqslant 0.1$ (Bozóki et al., 2013; Bozóki and Rapcsak, 2008; Monsuur, 1997). Consequently, a number of alternative methods have been suggested to address these drawbacks. Some of these methods are discussed below.

\subsubsection{Distance-based Measure}

Crawford (1987) proposed the logarithmic residual mean square as a natural measure of consistency 
whilst proposing the geometric mean (GM) method. Aguaron and Moreno-Jimenez (2003) formalized the logarithmic residual mean square, termed the Geometric Consistency Index (GCI), as:-

$$
G C I=\frac{2}{n(n-1)} \sum_{i=1}^{n-1} \sum_{j>i}^{n}\left(\log \left(a_{i j}\right)-\log \left(\frac{w_{i}}{w_{j}}\right)\right)^{2}
$$

where $w$ is a priority vector estimated using the GM method. They compared GCI to CR, and proposed threshold values for $G C I$ equivalent to $C R$.

\subsubsection{Measuring the Worst Error}

Koczkodaj (1993) described CR as a global measure that lacks a proper justification for its use. He proposed a measure, termed Consistency Measure (CM), in part to eliminate the drawbacks of CR, such as the inability to identify inconsistent judgments and its arbitrary threshold value of $C R \leqslant 0.1$.

$\mathrm{CM}$ is calculated by considering a set of three judgments (triple) from $J$ at a time. The value of $\mathrm{CM}$ is chosen from the most inconsistent triple amongst all possible combinations i.e.

$$
C M=\max _{i \neq j \neq k \neq i}\left(\overline{C M}_{i, j, k}\right)
$$

where $\overline{C M}_{i, j, k}$ is the inconsistency for the triple $\left\{a_{i j}, a_{j k}, a_{i k}\right\}$ and is calculated using the following formula:-

$$
\overline{C M}_{i, j, k}=\min \left(\frac{\left|a_{i j}-a_{i k} a_{k j}\right|}{a_{i j}}, \frac{\left|a_{i j}-a_{i k} a_{k j}\right|}{a_{i k} a_{k j}}\right)
$$

CM has been shown to be useful for improving consistency by revising judgments (Koczkodaj and Szarek, 2010). However, similarly to the CR index, this measure cannot capture the ordinal inconsistency of the comparison judgments.

\subsubsection{Kendall's $\zeta$}

In order to measure ordinal inconsistency, Kendall (1955) introduced an ordinal Coefficient of Consistence, $\zeta$, for comparisons between elements with no preference equivalencies. Kendall's $\zeta$ is calculated using the following equation:-

$$
\zeta=1-\frac{L}{L_{\max }}
$$

where $L$ is the number of circular triads present in given PCM, and $L_{\max }$ is the maximum number of circular triads possible. The value of $L_{\max }$ is $\left(\mathrm{n}^{3}-\mathrm{n}\right) / 24$ for odd values of $n$, and $\left(\mathrm{n}^{3}-4 \mathrm{n}\right) / 24$ when $n$ is even, formulated 
in (Kendall, 1955).

Gass (1998) formulated the prioritization problem for PCs as a tournament ranking problem and calculated the total number of cyclic judgments as:-

$$
L=\frac{n(n-1)(2 n-1)}{12}-\frac{1}{2} \sum_{i} s_{i}^{2}
$$

where $s_{i}$ is the total number of wins claimed by $E_{i}$ (also termed the out-degree). It should be noted that this definition of $L$ does not support preference equivalence.

In order to include preference equivalence, Jensen and Hicks (1993) extended Kendall's work and proposed the generalized coefficient of consistence. They suggested it be used as a supplement to CR, in the presence of intransitive judgments. The number of circular triads considering preference equivalence is always greater than $L$ for obvious reasons.

\subsection{Critique}

From their definitions, it can be seen that the CR and CM measures of consistency do not take into account the ordinal inconsistency (or intransitivity) of the PCM. This is also true for GCI, which has a linear relationship with CR (Aguaron and Moreno-Jimenez, 2003). Bozóki and Rapcsak (2008) compared $\mathrm{CR}$ and $\mathrm{CM}$ with a conclusion that no single measure is adequate in all situations. They also questioned the CR work for using randomly generated PCMs as the reference point and, hence, considered this reference to be inappropriate for analyzing human-based decisions.

Generally, if the comparison matrices are ordinally consistent, most prioritization methods derive priorities with the same ranking, only with different intensities. If, however, the matrices are ordinally inconsistent (intransitive), there exists no priority vector satisfying all contradictory preferences. Therefore, different prioritization methods provide different ordinal rankings that partially correspond to the ordinal comparison judgments.

\section{Judgment Scales and Inconsistency}

Experiments have been performed to investigate how well inconsistency in a PCM is measured when different scales of measurement are used. The authors have used Monte-Carlo simulation for this purpose. Monte-Carlo experiments are based on repeated random sampling to compute results that are usually not achieved through a deterministic algorithm. Such methods are useful to model phenomena with significant 


\begin{tabular}{rccccccccc}
\hline Linear & 1 & 2 & 3 & 4 & 5 & 6 & 7 & 8 & 9 \\
\hline Balanced & 1 & 1.111 & 1.125 & 1.143 & 1.167 & 1.20 & 1.25 & 1.333 & 1.5 \\
Geometric & 1 & 2 & 4 & 8 & 16 & 32 & 64 & 128 & 256 \\
Inverse & 1 & 1.125 & 1.286 & 1.5 & 1.8 & 2.25 & 3.0 & 4.5 & 9.0 \\
Logarithmic & 1 & 1.585 & 2 & 2.322 & 2.585 & 2.807 & 3 & 3.17 & 3.322 \\
Power & 1 & 4 & 9 & 16 & 25 & 36 & 49 & 64 & 81 \\
Root & 1 & 1.414 & 1.732 & 2 & 2.236 & 2.449 & 2.646 & 2.828 & 3 \\
\hline *
\end{tabular}

Table 2: The sets of values used for each judgment scale

uncertainty in inputs. Monte-Carlo methods have often been used previously for analyzing PCMs (Seaman et al., 1991; Herman and Koczkodaj, 1996; Koczkodaj, 1998; Ishizaka and Lusti, 2006).

PCMs were randomly generated using the sets of values given in Table 2. Each scale consists of equivalence (1), dominance $(>1)$ and their reciprocal values. For each $n$ ranging from 3 to 6,50000 PCMs were generated by randomly picking the values from the selected scale. The CC of each PCM was measured using $\mathrm{CR}$ while the $\mathrm{OC}$ was measured with the number of circular triads, $L$.

The results from these experiments are discussed below.

\subsection{Experimental Results}

The percentage of the acceptable PCMs found for each scale is shown in Table 3. Considering the linear scale of 1 to 9 , the percentage of acceptable PCMs decreases with the increase in $n$ i.e. $22.21 \%$ for $n=3$, $3.36 \%$ for $n=4$ and only $0.26 \%$ for $n=5$.

The geometric and power scales are least likely to produce acceptable PCMs. In the performed experiments, no PCM was found acceptable for $n=5$ when using the power scale.

For the inverse and root scales, the percentage decreases monotonically as the value of $n$ is increased. The possibility of having an acceptable PCM remains around $60 \%$ when using the logarithmic scale and is invariant to $n$. The chances of obtaining an acceptable PCM with the balanced scale are higher than with all the other tested scales and goes even higher for greater values of $n$ (see Table 3). When PCMs were randomly generated for $n=5$ using the balanced scale, all had CR below $0.1(100 \%)$.

It is inferred from these results that the possibility of having an acceptable PCM is highly sensitive to the selected scale. In other words, the value of CR is sensitive to the measurement scale chosen for generating PCMs.

In order to investigate the use of $\mathrm{CR}$ for measuring $\mathrm{OC}, 15000$ acceptable PCMs were generated for each scale. These matrices were then tested as to whether transitive $(L=0)$ or intransitive $(L \neq 0)$. The 


\begin{tabular}{cccccccc}
\hline $\mathbf{n}$ & Linear & Balanced & Geometric & Inverse & Log & Power & Root \\
\hline $\mathbf{3}$ & $22.21 \%$ & $94.59 \%$ & $12.97 \%$ & $41.78 \%$ & $67.61 \%$ & $10.10 \%$ & $51.72 \%$ \\
$\mathbf{4}$ & $3.36 \%$ & $98.88 \%$ & $0.47 \%$ & $18.37 \%$ & $58.71 \%$ & $0.29 \%$ & $29.91 \%$ \\
$\mathbf{5}$ & $0.26 \%$ & $99.93 \%$ & $0.01 \%$ & $7.34 \%$ & $58.15 \%$ & $0.00 \%$ & $14.64 \%$ \\
\hline
\end{tabular}

Table 3: Percentage of acceptable PCMs in randomly generated matrices

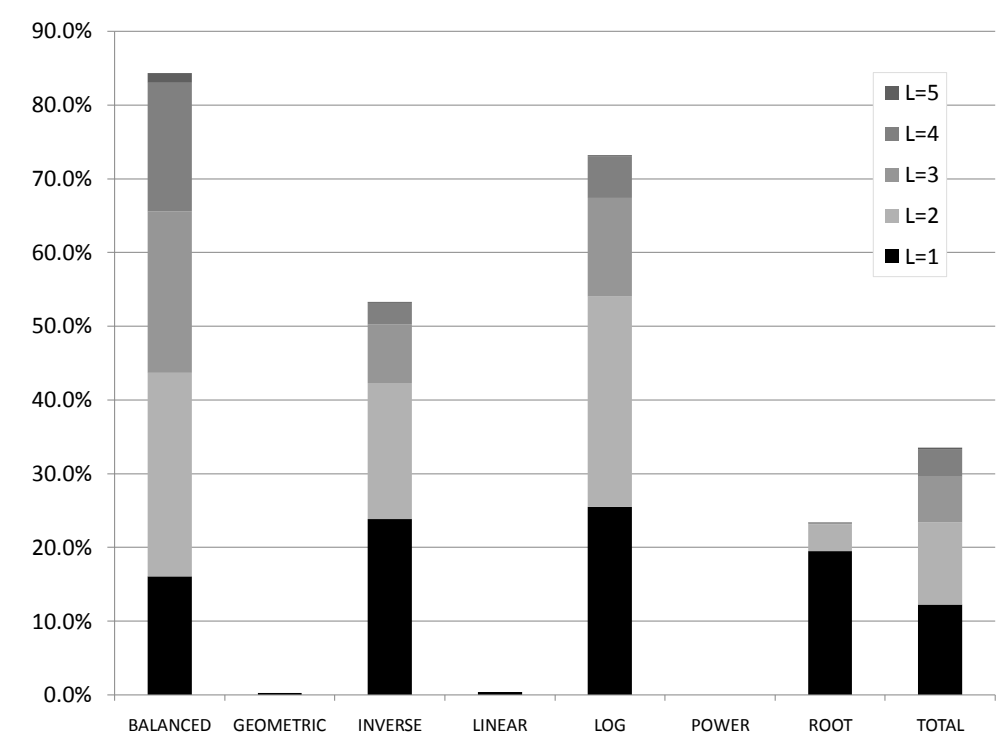

Figure 1: Percentage of IAPs found in acceptable PCMs

intransitive acceptable PCMs (IAPs) were then counted for each scale. The results for $n=5$ are shown in Fig. 1. Similar results were found for other values of $n$.

The percentage of IAPs was found to be very low for the linear and geometric scales (below 1\%). No IAP was generated for the power scale. The balanced scale produced the highest percentage of IAPs, followed by the logarithmic scale. When the inverse scale was used, more than half $(53.3 \%)$ of the generated PCMs were found to be intransitive. Similarly, 23.4\% of acceptable PCMs were found to be intransitive when using the root scale.

From the results given above, it is concluded that intransitive PCMs are not always rejected by Saaty's criterion of $C R \leqslant 0.1$.

\section{Congruence}

Considering the CC test between $E_{i}$ and $E_{j}$ i.e. $a_{i j}=a_{i k} a_{k j}$ (for all $i, j, k$ ), if the DM's judgments are cardinally inconsistent, then we obtain at least one indirect judgment $a_{i k} a_{k j} \neq a_{i j}$ for some $i, j$ and $k$ where $i \neq k \neq j$. Fig. 2 illustrates this concept by locating direct and indirect judgments together on a judgment 


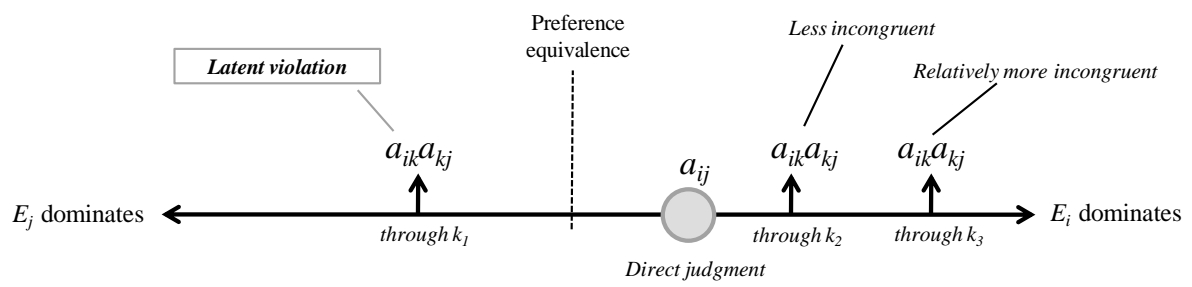

Figure 2: The indirect judgments $\left(a_{i k} a_{k j}\right)$ on a judgment scale

scale. Two of the indirect judgments in Fig. 2 suggest preference ratios higher than the direct judgment, while the third one (on the left) suggests a preference reversal (to be discussed in Section 5). When a DM provides a complete set of judgments in $J=\left\{a_{i j}\right\}$, a total of $\frac{n(n-1)(n-2)}{2}$ indirect judgments can be inferred.

Considering the dispersion of indirect judgments across the measurement scale, the deviation between $a_{i j}$ and the indirect judgment $a_{i k} a_{k j}$ can be formulated in a variety of ways, such as:-

$$
\begin{aligned}
\delta\left(a_{i j}, a_{i k} a_{k j}\right) & =\left|a_{i j}-a_{i k} a_{k j}\right| \\
\delta\left(a_{i j}, a_{i k} a_{k j}\right) & =\left(a_{i j}-a_{i k} a_{k j}\right)^{2} \\
\delta\left(a_{i j}, a_{i k} a_{k j}\right) & =\left|\frac{a_{i j}-a_{i k} a_{k j}}{a_{i j}}\right| \\
\delta\left(a_{i j}, a_{i k} a_{k j}\right) & =\left(\frac{a_{i j}-a_{i k} a_{k j}}{a_{i j}}\right)^{2} \\
\delta\left(a_{i j}, a_{i k} a_{k j}\right) & =\left|\log a_{i j}-\log \left(a_{i k} a_{k j}\right)\right| \\
\delta\left(a_{i j}, a_{i k} a_{k j}\right) & =\left(\log a_{i j}-\log \left(a_{i k} a_{k j}\right)\right)^{2}
\end{aligned}
$$

As multiple indirect judgments are possible for $n \geq 4$, the deviations need to be aggregated in order to define a measure of congruence, $\theta_{i j}$ for each judgment:-

$$
\theta_{i j}=\frac{1}{K_{i j}} \sum_{k} \delta\left(a_{i j}, a_{i k} a_{k j}\right) \forall\left\{a_{i j}, a_{i k}, a_{k j}\right\} \subseteq J
$$

where $k \neq i \neq j \neq k$ and $K_{i j}$ is the total number of indirect paths available between $E_{i}$ and $E_{j}$. When a complete set of judgments is available, the value of $K_{i j}$ is equal to $(n-2)$ for all judgments. In the case of an incomplete set of judgments, the values of $K_{i j}$ may have different values for different judgments.

The aggregation can also be formulated in other ways, for example, $\theta_{i j}$ can be taken as the worst deviation 
among all the indirect judgments i.e.

$$
\theta_{i j}=\max _{k}\left(\delta\left(a_{i j}, a_{i k} a_{k j}\right) \forall\left\{a_{i j}, a_{i k}, a_{k j}\right\} \subseteq J\right)
$$

or the geometric mean of all the indirect judgments i.e.

$$
\theta_{i j}=\left(\prod_{k} \delta\left(a_{i j}, a_{i k} a_{k j}\right)\right)^{\frac{1}{K_{i j}}} \forall\left\{a_{i j}, a_{i k}, a_{k j}\right\} \subseteq J
$$

It is clear that a single global value measuring inconsistency, whether cardinal or ordinal, is insufficient to locate all the inconsistent judgments present in $J$. Although Koczkodaj's CM has the property of detecting the most inconsistent judgment, it fails to describe the contribution of other judgments to the overall inconsistency. It is, therefore, proposed to measure inconsistency with the help of these individual congruence values for a given set of judgments.

For example, consider the following set of judgments:-

$$
\begin{aligned}
J_{1}: \quad a_{12} & =\frac{7}{4}, a_{13}=\frac{3}{4}, a_{14}=\frac{5}{2}, a_{15}=\frac{7}{4} \\
a_{23} & =\frac{3}{4}, a_{24}=\frac{9}{4}, a_{25}=\frac{9}{4} \\
a_{34} & =\frac{3}{4}, a_{45}=\frac{3}{4} \\
a_{45} & =\frac{5}{8}
\end{aligned}
$$

The set of judgments $J_{1}$ can be used to construct the following PCM:-

$$
A_{1}=\left[\begin{array}{ccccc}
1 & \frac{7}{4} & \frac{3}{4} & \frac{5}{2} & \frac{7}{4} \\
\frac{4}{7} & 1 & \frac{3}{4} & \frac{9}{4} & \frac{9}{4} \\
\frac{4}{3} & \frac{4}{3} & 1 & \frac{3}{4} & \frac{3}{4} \\
\frac{2}{5} & \frac{4}{9} & \frac{4}{3} & 1 & \frac{5}{8} \\
\frac{4}{7} & \frac{4}{9} & \frac{4}{3} & \frac{8}{5} & 1
\end{array}\right]
$$

The value of $\mathrm{CR}$ for $A_{1}$ is equal to 0.083 and, therefore, $A_{1}$ is considered to be acceptable in AHP terms. 
A congruence matrix for $A_{1}$ can be calculated using (14) and (12) as follows:-

$$
\theta=\left[\begin{array}{ccccc}
- & 0.61 & 1.06 & 0.69 & 0.69 \\
& - & 1.11 & 0.77 & 0.89 \\
& & - & 1.12 & 1.00 \\
& & & - & 0.35 \\
& & & & -
\end{array}\right]
$$

where the values for self-comparison and reciprocal judgments are not shown, being redundant. The use of logarithmic deviations here removes the possibility of having different values in the upper and lower triangles of the matrix. The values in $\theta$ can be sorted in descending order as follows:-

$$
\begin{array}{cccccccccc}
\theta_{34} & \theta_{23} & \theta_{13} & \theta_{35} & \theta_{25} & \theta_{24} & \theta_{14} & \theta_{15} & \theta_{12} & \theta_{45} \\
1.12 & 1.11 & 1.06 & 1.00 & 0.89 & 0.77 & 0.69 & 0.69 & 0.61 & 0.35
\end{array}
$$

The judgment, $a_{34}$ is found to be most inconsistent $\left(\theta_{34}=1.12\right)$, while $a_{45}$ is considered the most consistent one. The two judgments $a_{14}$ and $a_{15}$ are found to be equally inconsistent (i.e. $\theta_{14}=\theta_{15}=0.69$ ). Similarly, this can be calculated for other PCMs generated using different scales.

It is emphasized here that the values of $\theta_{i j}$ in this example are calculated using logarithmic deviation between direct and indirect judgments, as given in (12). The use of other definitions may produce different results and the appropriateness of each definition may be further investigated.

\subsection{Overall Congruence}

If need be, the set of values $\left\{\theta_{i j}\right\}$ can further be used to derive a single global measure - the overall congruence - through an aggregation procedure, for example:-

$$
\Theta=\frac{1}{m} \sum \theta_{i j} \forall a_{i j} \in J
$$

where $m$ is the total number of judgments acquired in $J$. Like $\theta_{i j}$, the overall congruence, $\Theta$ can also be formulated using other approaches, such as choosing the worst deviation amongst all the measured ones i.e.

$$
\Theta=\max \left(\theta_{i j} \forall a_{i j} \in J\right)
$$

It should be highlighted that $\Theta$ can have many definitions, depending on the way individual congruence values are aggregated and the formulation used for $\delta$. For example, the overall congruence value for $J_{1}$ (in 
the above example) will be equal to $\Theta=0.828$ when using (16), or $\Theta=1.12$ when using (17).

Recall: the value of $\mathrm{CR}$ is sensitive to the measurement scale and is only suitable for a linear scale of 1 to 9. In contrast, the congruence measure is applicable to any scale in a straight-forward manner. However, the threshold of acceptance for each scale may differ and requires investigation.

\subsection{1. $C M$ and GCI: Examples of Congruence}

As discussed earlier, Aguaron and Moreno-Jimenez (2003) formalized GCI as the aggregated logarithmic residuals, defined in (3). It can be shown that the GCI measure can be formulated as a congruence measure by using logarithmic squared deviations (given in (13) for $\delta$ ), (14) for $\theta$ and (16) for $\Theta$.

Similarly, CM can also be formulated as a congruence measure. As discussed earlier, the value of CM is chosen from the most inconsistent triple amongst all possible combinations of indirect judgments (Koczkodaj and Szarek, 2010). On carefully observing the multiple definitions of $\delta, \theta$ and $\Theta$, it can be concluded that CM can be obtained with $\delta$ in (10), $\theta$ in (15), and $\Theta$ defined in (17).

In other words, it can be concluded that $\mathrm{CM}$ and GCI can be seen as the two possible aggregates for inconsistencies present in individual judgments.

\subsection{Incomplete Sets of Judgments}

Harker (1987) investigated an incomplete set of judgments, where DMs are allowed to respond with "don't know" or "not sure" to some judgments. It has been highlighted that the probability of acquiring incomplete PCs increases as n increases (Fedrizzi and Giove, 2007; Fedrizzi, 2012). The proposed congruence measure is applicable to incomplete sets of PC judgments without modification, unlike CR which requires a complete set of PC judgments.

Consider the following incomplete form of $A_{1}$ :-

$$
\check{A}_{1}=\left[\begin{array}{ccccc}
1 & \frac{7}{4} & - & \frac{5}{2} & \frac{7}{4} \\
\frac{4}{7} & 1 & \frac{3}{4} & \frac{9}{4} & \frac{9}{4} \\
- & \frac{4}{3} & 1 & \frac{3}{4} & \frac{3}{4} \\
\frac{2}{5} & \frac{4}{9} & \frac{4}{3} & 1 & \frac{5}{8} \\
\frac{4}{7} & \frac{4}{9} & \frac{4}{3} & \frac{8}{5} & 1
\end{array}\right]
$$

Here, the DM has not provided the judgment $a_{13}$ and, therefore, the value of CR cannot be calculated for the incomplete PCM. In contrast, congruence values for the provided judgments are shown below:- 


$$
\check{\theta}=\left[\begin{array}{ccccc}
- & 0.42 & - & 0.19 & 0.31 \\
& - & 0.92 & 0.77 & 0.89 \\
& & - & 0.62 & 0.62 \\
& & & - & 0.35 \\
& & & & -
\end{array}\right]
$$

The most inconsistent judgment is found to be $a_{23}$ with the congruence value of $\check{\theta}_{23}=0.92$. The judgment $a_{25}$ is found to be the next most inconsistent while $a_{45}$ is still considered to be the most consistent one. The overall congruence for $\check{A}_{1}$ is $\check{\Theta}=0.51$.

\subsection{Outlier Detection}

The set of congruence values, $\theta_{i j}$, can be used to help spot the outlier with the highest deviation value. In order to identify an outlier with the help of congruence, consider the following example:-

$$
A_{2}=\left[\begin{array}{ccccc}
1 & 2 & 4 & 8 & \frac{1}{3} \\
\frac{1}{2} & 1 & 2 & 4 & 8 \\
\frac{1}{4} & \frac{1}{2} & 1 & 2 & 4 \\
\frac{1}{8} & \frac{1}{4} & \frac{1}{2} & 1 & 2 \\
3 & \frac{1}{8} & \frac{1}{4} & \frac{1}{2} & 1
\end{array}\right]
$$

The congruence matrix for $A_{2}$ can be calculated using (12) and (14) as follows:-

$$
\theta=\left[\begin{array}{ccccc}
- & 1.29 & 1.29 & 1.29 & 3.87 \\
& - & 0 & 0 & 1.29 \\
& & - & 0 & 1.29 \\
& & & - & 1.29 \\
& & & & -
\end{array}\right]
$$

The value of $\theta_{15}=3.87$ shows that $a_{15}$ is the least congruent with other judgments. The three judgments of $a_{23}, a_{34}$ and $a_{24}$ are found to be perfectly consistent. All the other judgments make an equal contribution to the overall inconsistency. As there exists a single judgment that shows the highest deviation, this suggests the presence of an outlier i.e. $a_{15}$. 


\subsection{Consistency Deadlock}

Consider another example of inconsistent PCM as follows:-

$$
A_{3}=\left[\begin{array}{ccccc}
1 & 2 & 2 & 2 & 2 \\
\frac{1}{2} & 1 & 2 & 2 & 2 \\
\frac{1}{2} & \frac{1}{2} & 1 & 2 & 2 \\
\frac{1}{2} & \frac{1}{2} & \frac{1}{2} & 1 & 2 \\
\frac{1}{2} & \frac{1}{2} & \frac{1}{2} & \frac{1}{2} & 1
\end{array}\right]
$$

The congruence matrix for $A_{3}$ is as follows:-

$$
\theta=\left[\begin{array}{ccccc}
- & .69 & .69 & .69 & .69 \\
& - & .69 & .69 & .69 \\
& & - & .69 & .69 \\
& & & - & .69 \\
& & & & -
\end{array}\right]
$$

This matrix tells a completely different story: all given judgments are found to be equally inconsistent. This type of situation can be termed "consistency deadlock" as no improvement can be suggested. A single global measure of consistency (e.g. CR) cannot describe the presence of consistency deadlock. In such cases, any of the provided judgments can be selected for revision.

\section{Dissonance}

The concept of indirect judgments can also be used to calculate OC, based on the ordinal violation between a given judgment and the corresponding indirect judgments. The preference relation $C_{i} \rightarrow C_{j}$ (implying $a_{i j}>1$ ) should enforce $a_{i k} a_{k j}>1$ for all $k=1,2, \ldots, n$. Therefore, whenever an indirect judgment contradicts this preference relation, a latent violation occurs. The concept of latent violation can be visualized in Fig. 2, which shows a violation of $a_{i j}$ by an indirect judgment through $k_{1}$. This is mathematically formulated as:-

$$
\psi_{i j}=\frac{1}{K_{i j}} \sum_{k} \operatorname{step}\left(-\log a_{i j} \log a_{i k} a_{k j}\right) \forall\left\{a_{i j}, a_{i k}, a_{k j}\right\} \subseteq J
$$


where $i \neq k \neq j \neq i$ and the step function is defined as:-

$$
\operatorname{step}(x)= \begin{cases}1 & \text { if } x>0 \\ 0 & \text { otherwise }\end{cases}
$$

The set of values $\psi_{i j}$ construct a dissonance matrix that can supplement the congruence matrix in order to spot the most inconsistent judgments. The term proposed here relates to the notion of cognitive dissonance i.e. the holding of two contradictory beliefs simultaneously.

It is proposed to calculate $\mathrm{OC}$ as the average of all the elements in $\left\{\psi_{i j}\right\}$ as overall dissonance $(\Psi)$ :-

$$
\Psi=\frac{1}{m} \sum \psi_{i j} \forall a_{i j} \in J
$$

where $m$ is the total number of judgments acquired in $J$. The average is proposed instead of the aggregation to remove its dependence on $n$.

Although the values of $C R, C M$ and $\Theta$ measure the level of inconsistency in judgments, $\Psi$ uniquely measures ordinal inconsistency (as with Kendall's $\zeta$ ), suggesting a number of possible preference reversals. This dissonance can be used to detect priority violations, as illustrated through examples in the next section.

The calculation of congruence matrix, $\left[\theta_{i j}\right]$, has been useful to analyze cardinal inconsistency. The use of dissonance matrix, $\left[\psi_{i j}\right]$, can further supplement the visualization of ordinal inconsistency. This is explained here with the help of examples already discussed above.

Considering $A_{1}$ again, the dissonance matrix is obtained using (18) as follows:-

$$
\psi=\left[\begin{array}{ccccc}
- & 0.33 & 1.00 & 0.33 & 0.33 \\
& - & 0.67 & 0.33 & 0.33 \\
& & - & 1.00 & 0.67 \\
& & & - & 0.35 \\
& & & & -
\end{array}\right]
$$

where transpose elements are not shown, as they are redundant. The value of $\psi_{34}=1$ suggests $a_{34}$ to be completely dissonant with all the related judgments in $A_{1}$. The judgment of $a_{34}$, which was declared to be the most inconsistent, has also been found to be the most dissonant.

Consider another interesting example where the most inconsistent and the most dissonant judgments are different, as follows:- 


\begin{tabular}{ccccccccccc}
\hline & $a_{12}$ & $a_{13}$ & $a_{15}$ & $a_{23}$ & $a_{35}$ & $a_{14}$ & $a_{24}$ & $a_{45}$ & $a_{34}$ & $a_{25}$ \\
\hline$\theta \Longrightarrow$ & 2.10 & 1.87 & 1.66 & 1.60 & 1.53 & 1.43 & 1.33 & 1.16 & 1.13 & 0.95 \\
$\psi \Longrightarrow$ & 0.33 & 0.67 & 0.33 & 0.67 & 0 & 1 & 0.67 & 0.33 & 0.67 & 0 \\
\hline
\end{tabular}

Table 4: Individual congruence \& dissonance values for the judgments in $A_{4}$

$$
A_{4}=\left[\begin{array}{ccccc}
1 & 9 & \frac{3}{4} & \frac{3}{4} & 9 \\
\frac{1}{9} & 1 & \frac{5}{4} & \frac{3}{4} & 4 \\
\frac{4}{3} & \frac{4}{5} & 1 & 2 & \frac{4}{3} \\
\frac{4}{3} & \frac{4}{3} & \frac{1}{2} & 1 & 3 \\
\frac{1}{9} & \frac{1}{4} & \frac{3}{4} & \frac{1}{3} & 1
\end{array}\right]
$$

The two consistency measures, calculated for each judgment in $A_{4}$, are given in Table 4 where the judgments are arranged with the values of $\theta_{i j}$ in descending order. The value $\psi_{14}=1$ for $a_{14}$ implies that no judgment in $A_{4}$ supports the order of preference suggested by $a_{14}$. Whilst the most dissonant judgment is $a_{14}$ (i.e. $\psi_{14}=1$ ), the most inconsistent is found to be $a_{12}$ according to the CC measure. This paradox has generated the question of whether $\mathrm{CC}$ should be given precedence over $\mathrm{OC}$. An answer to this question remains an area of further investigation.

\section{Approaches for Improving Consistency}

When both the ordinal and the cardinal inconsistency in PC judgments are found, the next obvious step is to improve the consistency prior to the process of prioritization. Different techniques have been introduced to detect and rectify inconsistency, either by using a local operator or a matrix-based global operation (Zeshui and Cuiping, 1999; Cao et al., 2008; Ergu et al., 2011; Siraj et al., 2012). The improvements can be done manually by asking the DM to revise the judgments, or alternatively, improvements can be done using an automated approach.

\subsection{Manual Revision of Judgments}

In situations where the provided judgments are allowed to be revised, it is suggested that the DM is offered both $\theta_{i j}$ and $\psi_{i j}$. For example, consider the PCM, $A_{4}$ in the form of a table, shown in Fig. 3a. The DM is supposed to manually revise by changing the values of $a_{i j}$.

The two consistency measures, $\theta$ and $\psi$, are shown along with the original judgments in Fig. 3a. The values of $\theta_{i j}$ are shown above $a_{i j}$ while $\psi_{i j}$ is shown below $a_{i j}$ in each cell. In practical situations, the DM 


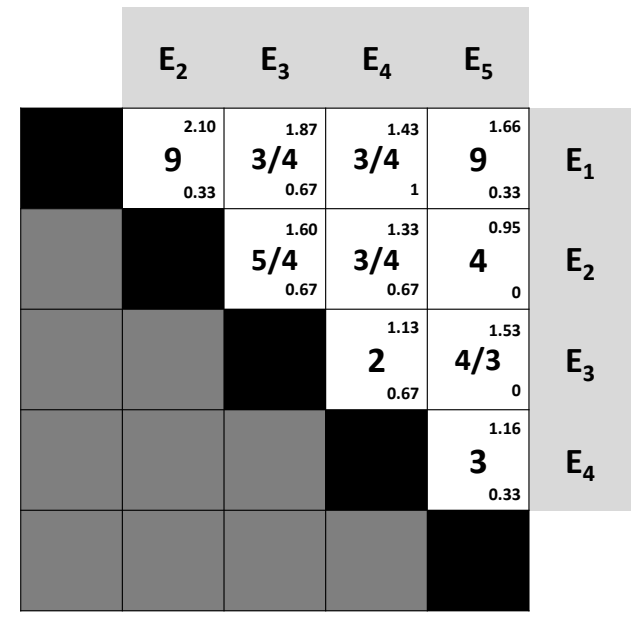

(a) as numeric values

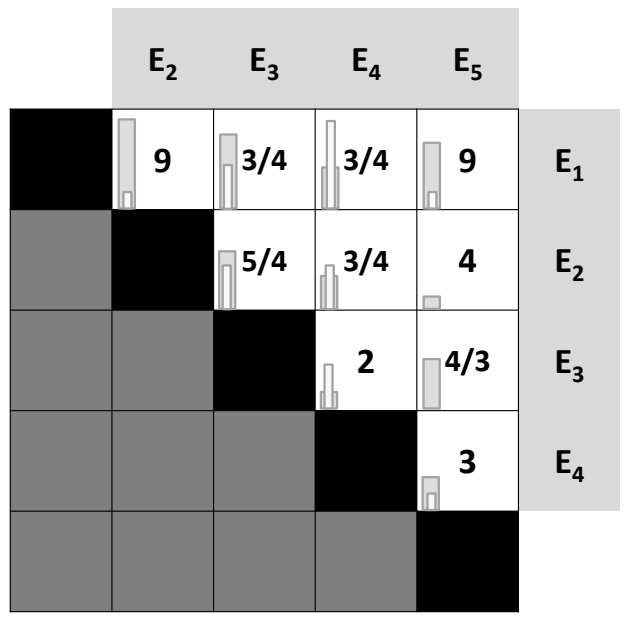

(b) as a graphical aid

Figure 3: Offering $\theta$ and $\psi$ for $A_{4}$

may find it difficult to make reasonably correct assessments as several numeric values on the table may cause information overload.

\subsection{1. $\theta$ and $\psi$ as a Graphical Aid}

A better approach is to visualize the consistency of each judgment graphically as a bar graph. Fig. 3b shows this approach for $A_{4}$, where congruence is shown as a grey bar with each judgment and dissonance is shown as a white bar. A DM can easily spot the most inconsistent judgment $\left(a_{12}=9\right)$ to manually improve its value. When $\mathrm{OC}$ has precedence, the value of $a_{14}$ should be rectified first as it is ordinally the most inconsistent.

This graphical approach is useful to spot both outliers and the phenomenon of consistency deadlock. Consider the two matrices $A_{2}$ and $A_{3}$, already discussed in Section 4. Fig. 4a highlights the presence of an outlying judgment in $A_{2}$ where $a_{15}$ is shown to be the most inconsistent, both cardinally and ordinally. Similarly, the phenomenon of consistency deadlock for $A_{3}$ is visually represented in Fig. 4b. All the judgments in $A_{3}$ are shown here to be equally responsible for inconsistency. There is no ordinal inconsistency present in $A_{3}$.

\subsection{Reducing Inconsistency using an Automated Approach}

There exist situations in practice where acquired data cannot be either changed or not allowed to be changed and, hence, manual revision of judgments is not possible. One way to address this is to automatically 


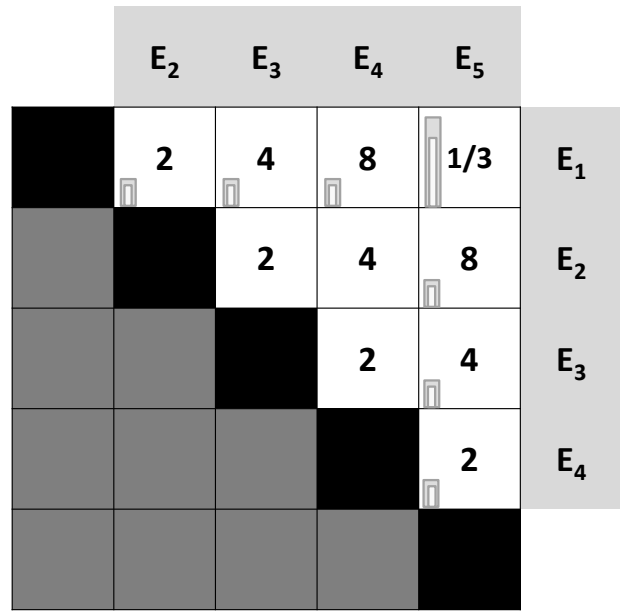

(a) Judgment $a_{15}$ as an outlier in $A_{2}$

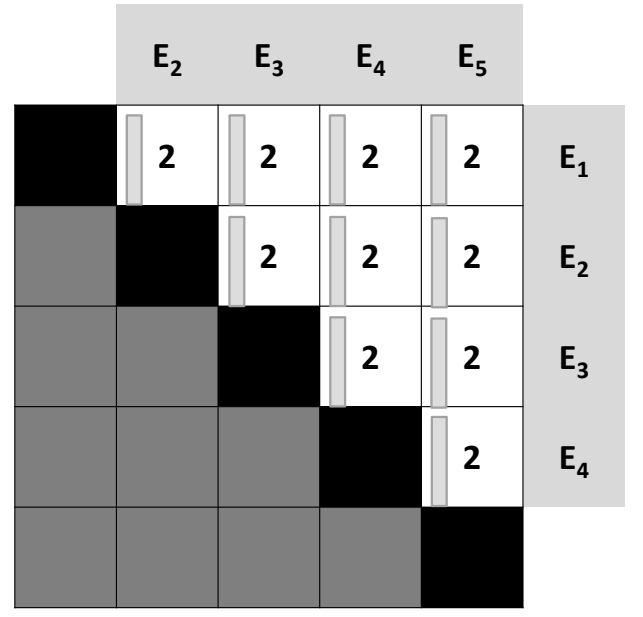

(b) Consistency deadlock in $A_{3}$

Figure 4: Visualizing the phenomena of outlier detection and consistency deadlock

remove inconsistent judgments with minimal deviation from the initial judgments. This can be achieved using the congruence matrix; choosing the judgment with the highest value of $\theta$. When a set of judgments is ordinally inconsistent, the use of the dissonance matrix proves supplemental, suggesting a two-step process i.e. firstly, choose the set of judgments with the highest value of $\psi$ and then, from these candidates, select the judgment with the highest value of $\theta$.

In the presence of an outlier, a revised value for the blamed judgment can be calculated as:-

$$
\tilde{a}_{i j}=\left(\prod_{k} a_{i k} a_{k j}\right)^{\frac{1}{n-2}}
$$

where $i \neq k \neq j$. Another way to calculate a consistent value is to use the arithmetic mean i.e. $a_{i j}=$ $\frac{1}{n-2} \sum_{k} a_{i k} a_{k j}$ where $i \neq k \neq j$. The use of the arithmetic or geometric mean is also an area for further investigation.

In the case of ordinal inconsistency, the preference order of the most inconsistent judgment needs to be reversed. The suggested averaging process may not work in the presence of ordinal inconsistency. Considering the minimum deviation from the original value, a revised value can be calculated as:-

$$
\tilde{a}_{i j}= \begin{cases}0.99 & \text { if } a_{i j}>1 \\ 1.01 & \text { if } a_{i j}<1\end{cases}
$$


Consider $A_{2}$ for example; the value for $a_{15}$ can be changed from $\frac{1}{3}$ to 16 using (20). If minimal change is to be expected, then the value of $a_{15}$ should be changed to 1.01 using (21). Considering the phenomenon of consistency deadlock, as all the judgments in $A_{3}$ are equally inconsistent; the only possible improvement is to randomly select a judgment and replace its value using (20) which is equal to 4 .

In the case of $A_{1}, a_{12}$ is cardinally the most inconsistent and the suggested correction is $\left(a_{13} a_{32} a_{14} a_{42} a_{15} a_{52}\right)^{\frac{1}{3}}=$ 1.1. If ordinal inconsistency has priority, then $a_{14}$ should instead be corrected and the suggested value for $a_{14}$ is 1.01 using (21).

Holsztynski and Koczkodaj (1996) proved that removing local inconsistencies incrementally will improve the overall consistency of PCMs. The same proof justifies convergence of the improvement process using $\theta_{i j}$ and $\psi_{i j}$.

\section{Illustrative Examples}

To illustrate the concept of indirect judgments and the use of congruence and dissonance, four different types of PCMs are discussed below.

\subsection{Consistent PCM}

Consider an example of a consistent PCM given below:-

$$
A_{e x 1}=\left[\begin{array}{cccc}
1 & 2 & 4 & 12 \\
\frac{1}{2} & 1 & 2 & 6 \\
\frac{1}{4} & \frac{1}{2} & 1 & 3 \\
\frac{1}{12} & \frac{1}{6} & \frac{1}{3} & 1
\end{array}\right]
$$

The set of all indirect judgments calculated for $A_{e x 1}$ are identical to the direct judgments, therefore $\Theta=0$ and $\Psi=0$. The other consistency measures, CR and CM, also produce the same value, as expected. Hence, $A_{e x 1}$ contains a set of congruent judgments.

This can be visualized in Fig. 5a where each judgment lying in the upper triangular part of $A_{e x 1}$ is shown on a logarithmic scale. A circle in the figure depicts a direct judgment while indirect judgments are shown as an upwards arrow. All the indirect judgments in this case appear aligned with their direct judgment, following the consistency rule of $a_{i j}=a_{i k} a_{k j}$. Hence, all the prioritization methods generate the same weights i.e. $w=[0.5450 .2730 .1360 .045]$. 


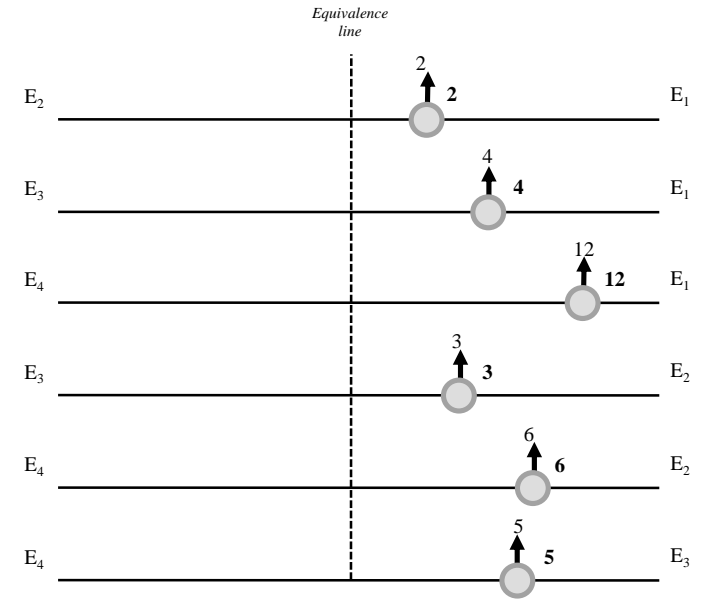

(a) $A_{e x 1}$ with consistent judgments

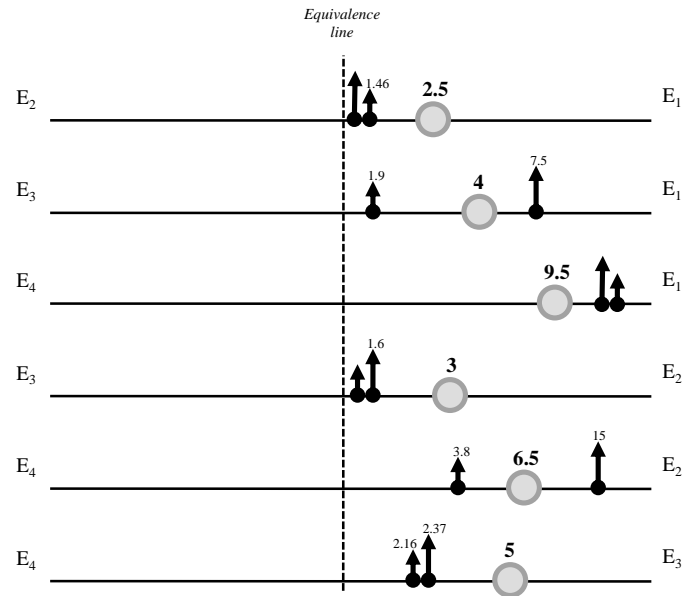

(b) $A_{\text {ex } 2}$ having inconsistent judgments

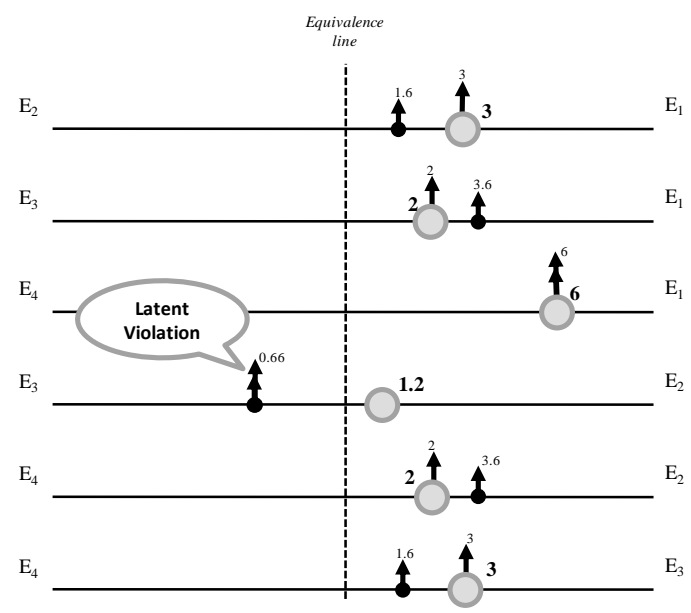

(c) $A_{\text {ex3 }}$ having transitive but dissonant judgments

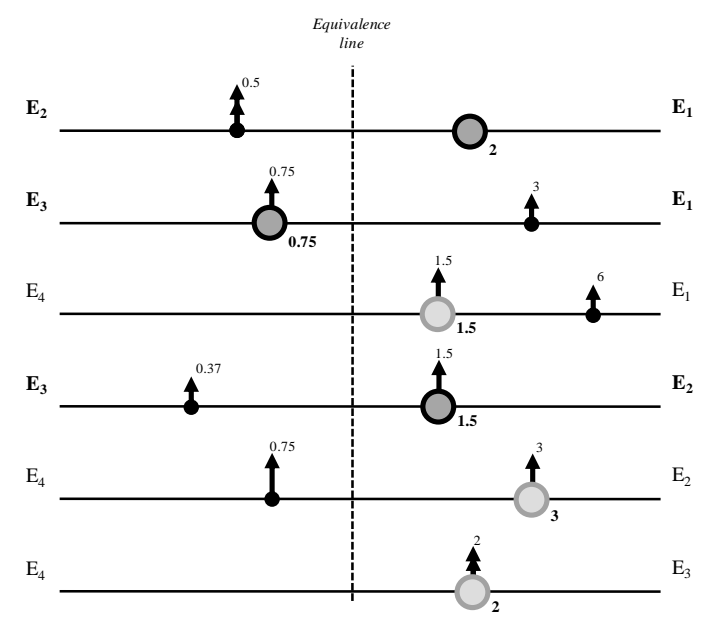

(d) $A_{\text {ex } 4}$ having intransitive set of judgments

Figure 5: Graphical views of the direct and indirect judgments for the illustrated examples 


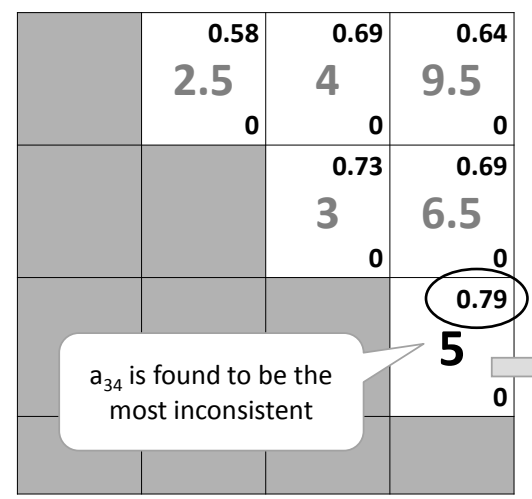

Initial Set of Judgments $C R=0.045, C M=0.567, \Theta=0.686$

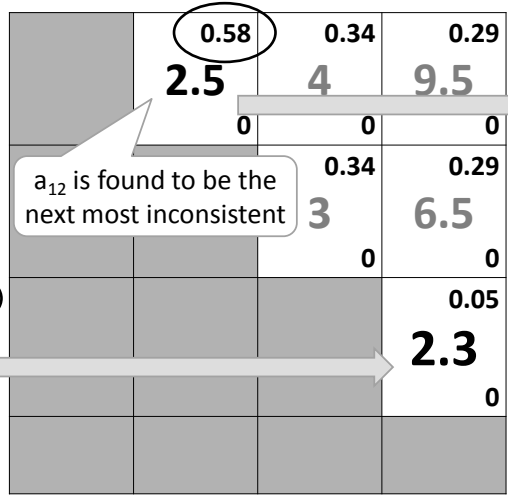

after Iteration 1 $C R=0.016, C M=0.467, \Theta=0.314$

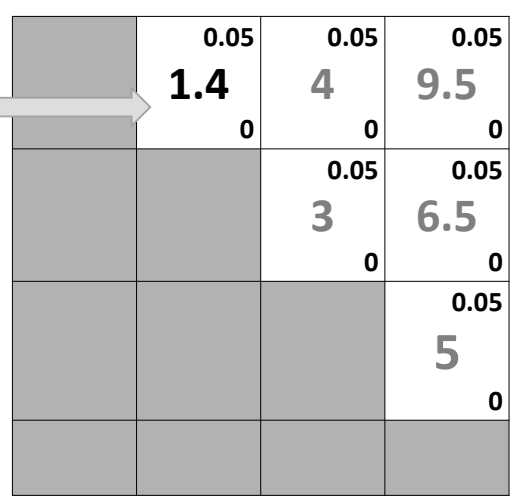

after Iteration 2

$C R=0.001, C M=0.045, \Theta=0.046$

Figure 6: Reducing Inconsistency in $A_{e x 2}$ using the Automated Approach

\subsection{Transitive PCM}

Consider the following inconsistent PCM with $C R<0.1$ and $L=0$ :-

$$
A_{e x 2}=\left[\begin{array}{cccc}
1 & 2 \frac{1}{2} & 4 & 9 \frac{1}{2} \\
\frac{2}{5} & 1 & 3 & 6 \frac{1}{2} \\
\frac{1}{4} & \frac{1}{3} & 1 & 5 \\
\frac{2}{19} & \frac{2}{13} & \frac{1}{5} & 1
\end{array}\right]
$$

Unlike $A_{e x 1}$, the indirect judgments in $A_{e x 2}$ are not equal to the direct ones, yet the order of preference dominance remains. As can be seen in Fig. 5b, no indirect judgment has caused latent violations implying

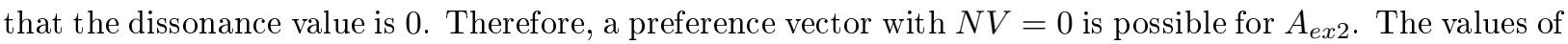
$\mathrm{CR}=0.045, \mathrm{CM}=0.567$ and $\Theta=0.686$ all suggest cardinal inconsistency among judgments, while $\Psi$ confirms that $A_{e x 2}$ is ordinally consistent.

Inconsistency in $A_{e x 2}$ can be improved using the automated approach as shown in Fig. 6 . The contribution of each judgment in $A_{e x 2}$ towards overall inconsistency is shown on the left of the figure, where $a_{34}$ is highlighted to be the most inconsistent. In the first iteration, the value of $a_{34}$ is updated using (20). This reduces the overall inconsistency from $\Theta=0.686$ to $\Theta=0.314$. During next iteration, the judgment $a_{12}$ is found to be the second most inconsistent and eventually been changed from $a_{12}=2.5$ to $a_{12}=1.4$ using (20). This step has further reduced the overall inconsistency from $\Theta=0.314$ to $\Theta=0.046$ (as shown on right side of Fig. 6). 


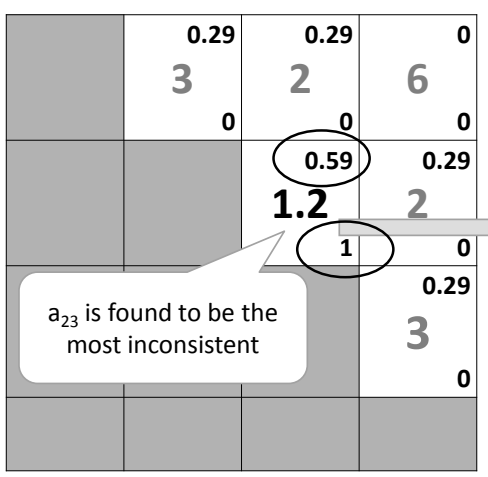

Initial Set of Judgments $C R=0.045, C M=0.567, \Theta=0.686$

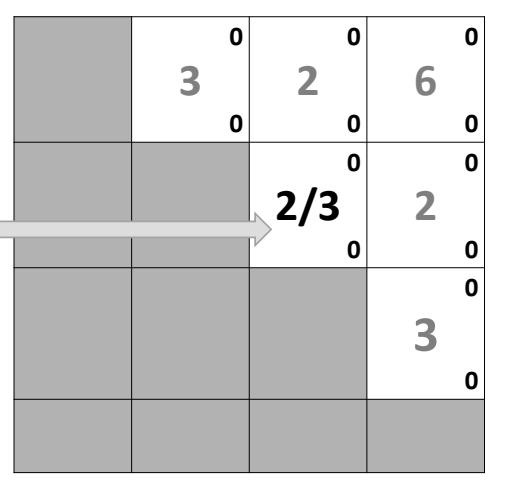

Iteration 1

$C R=0.0, C M=0.0, \Theta=0.0$

Figure 7: Reducing Inconsistency in $A_{e x 3}$ using the Automated Approach

\subsection{Transitive PCM with Latent Violations}

Another PCM with $C R<0.1$ and $L=0$ is considered below:-

$$
A_{e x 3}=\left[\begin{array}{cccc}
1 & 3 & 2 & 6 \\
\frac{1}{3} & 1 & 1 \frac{1}{5} & 2 \\
\frac{1}{2} & \frac{5}{6} & 1 & 3 \\
\frac{1}{6} & \frac{1}{2} & \frac{1}{3} & 1
\end{array}\right]
$$

$A_{\text {ex3 }}$ is included here to investigate second-order intransitivity. Hartvigsen (2005) pointed out that this comparison is transitive yet no method produces the ideal ranking. Considering the indirect judgments shown in Fig. 5c, we can easily identify latent violations between $E_{2}$ and $E_{3}$. The direct judgment for $a_{23}$ is 1.2 , however, the two indirect judgments suggest 0.66 , causing a priority violation whilst estimating weights. Although the values $C R=0.031, C M=0.555$ and $\Theta=0.405$ highlight judgment inconsistency, the only measure suggesting a possibility of a priority violation is $\Psi=0.167$.

Inconsistency in $A_{e x 3}$ can be improved using the automated approach as shown in Fig. 7. The congruence value of $\theta_{23}=0.59$ and the dissonance value of $\psi_{23}=1.0$ both suggest changing the value of $a_{23}$. The value of $a_{23}$ can be changed from 1.2 to $\frac{2}{3}$ using (20). This makes the matrix $A_{\text {ex } 3}$ (fully) consistent.

\subsection{Intransitive PCM}

An intransitive PCM $(L \neq 0)$ always contains dissonant judgments. This is illustrated below with the following intransitive PCM:- 


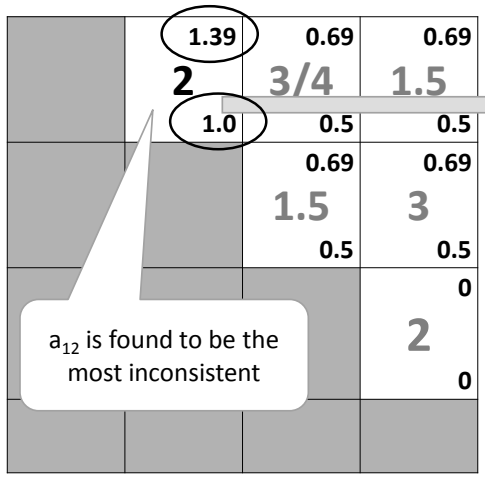

Initial Judgments $\mathrm{CR}=0.092 \mathrm{CM}=0.75 \Theta=0.693$

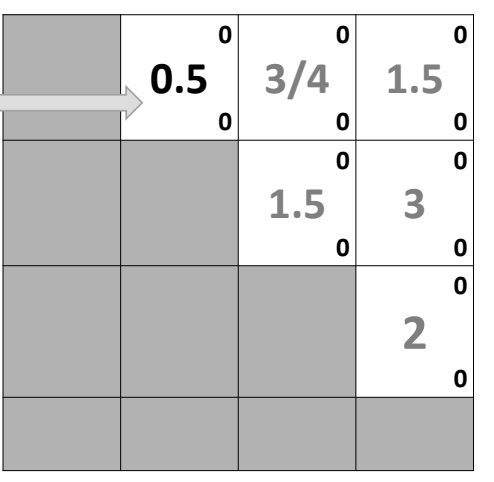

Iteration 1 $\mathrm{CR}=0.0 \mathrm{CM}=0.0 \Theta=0.0$

Figure 8: Reducing Inconsistency in $A_{e x 4}$ using Automated Approach

$$
A_{e x 4}=\left[\begin{array}{cccc}
1 & 2 & \frac{3}{4} & 1 \frac{1}{2} \\
\frac{1}{2} & 1 & 1 \frac{1}{2} & 3 \\
\frac{4}{3} & \frac{2}{3} & 1 & 2 \\
\frac{2}{3} & \frac{1}{3} & \frac{1}{2} & 1
\end{array}\right]
$$

There is a circular triad present in $A_{\text {ex } 4}$ i.e. $E_{1} \rightarrow E_{2} \rightarrow E_{3} \rightarrow E_{1}$. We discuss this example in detail with the help of Fig. 5d. The two indirect judgments for $a_{34}$ are perfectly consistent, while the judgment $a_{14}$ has one consistent and one inconsistent judgment. Each of the three judgments, $a_{13}, a_{23}$ and $a_{24}$, show one latent violation. Finally looking at $a_{12}$, we see that both indirect judgments are in conflict with the direct judgment. Thus the dissonance value $\phi_{12}$ is maximum (equals to 1 ), suggesting the judgment $a_{12}$ to be the outlier in this PCM.

Inconsistency in $A_{e x 4}$ can be improved using the congruence and dissonance matrices as shown in Fig. 8 . The congruence value of $\theta_{12}=1.39$ and the dissonance value of $\psi_{12}=1.0$ both suggest changing the value of $a_{12}$. The judgment $a_{12}$ can be updated from 2 to $\frac{1}{2}$ using (20). This makes the matrix $A_{\text {ex } 4}$ (fully) consistent as all the indirect judgments become aligned with the direct judgments after this revision.

The given examples have suggested measuring consistency as a pair of values i.e. $(\Theta, \Psi)$. Further investigation is required to explore the properties of these two consistency measures.

\subsection{Discussion}

The congruence measure requires a threshold value as with, for example, $C R \leqslant 0.1$ that declares a PCM to be acceptable. Such a threshold would also enable individual judgments to be declared to be acceptable or unacceptable. 
For purpose of demonstration, assume that the threshold value of 0.6 for congruence has been selected for the following two PCMs generated with the linear scale of 1 to 9:-

$$
\begin{aligned}
A_{1}= & {\left[\begin{array}{ccccc}
1 & 2 & 4 & 8 & \frac{1}{3} \\
\frac{1}{2} & 1 & 2 & 4 & 8 \\
\frac{1}{4} & \frac{1}{2} & 1 & 2 & 4 \\
\frac{1}{8} & \frac{1}{4} & \frac{1}{2} & 1 & 2 \\
3 & \frac{1}{8} & \frac{1}{4} & \frac{1}{2} & 1
\end{array}\right] } \\
A_{2}= & {\left[\begin{array}{ccccc}
1 & 2 & 4 & 8 & 9 \\
\frac{1}{2} & 1 & 2 & 4 & 8 \\
\frac{1}{4} & \frac{1}{2} & 1 & 2 & 4 \\
\frac{1}{8} & \frac{1}{4} & \frac{1}{2} & 1 & 2 \\
3 & \frac{1}{8} & \frac{1}{4} & \frac{1}{2} & 1
\end{array}\right] }
\end{aligned}
$$

The value of $\mathrm{CR}$ for $A_{1}$ is 0.55 suggesting that the PCM is unacceptable and the value of CR for $A_{2}$ is 0.009 that qualifies $A_{2}$ as an acceptable PCM. However, the use of CR does not reveal the sources of inconsistency in these PCMs.

The congruence matrices for $A_{1}$ and $A_{2}$ are shown below:-

$$
\begin{aligned}
& A_{1}: \theta=\left[\begin{array}{ccccc}
- & 1.29 & 1.29 & 1.29 & \underline{\mathbf{3 . 8 7}} \\
& - & 0 & 0 & 1.29 \\
& & - & 0 & 1.29 \\
& & & - & 1.29 \\
& & & & -
\end{array}\right] \\
& A_{2}: \theta=\left[\begin{array}{ccccc}
- & & & & \\
0.19 & 0.19 & 0.19 & \underline{\mathbf{0 . 5 8}} \\
- & 0 & 0 & 0.19 \\
& & - & 0 & 0.19 \\
& & & - & 0.19 \\
& & & & -
\end{array}\right]
\end{aligned}
$$

The congruence measure detects $a_{15}$ to be an outlier in both PCMs. The value of $\theta_{15}=3.87$ for $A_{1}$ suggests that judgment $a_{15}$ is highly inconsistent. The value of $\theta_{15}=0.58$ for $A_{2}$ suggests that $a_{15}$ is less 
inconsistent in $A_{2}$ than in $A_{1}$.

The overall congruence, $\Theta$, for $A_{1}$ is equal to 1.16 using (16) (i.e. the average of the individual congruence values). The threshold of 0.6 suggests that the PCM is unacceptable as $\Theta>0.6$. Based on this threshold, only three judgments in $A_{1}$ are found to be acceptable i.e. $a_{23}, a_{34}$ and $a_{24}$.

$A_{2}$ has an overall congruence value of $\Theta=0.17$. The threshold of 0.6 declares $A_{2}$ to be an acceptable PCM. All the judgments in $A_{2}$ are also considered to be acceptable when using this threshold value.

As demonstrated above, a properly justified threshold value for congruence will offer increased utility in comparison to the threshold available for $\mathrm{CR}$. We consider this to be an important area for future work: finding an appropriate threshold value for the congruence measure to determine acceptable and unacceptable judgments in a PCM.

\section{Conclusions}

This paper has investigated a number of widely used consistency measures with the help of Monte-Carlo simulations. This has shown that a single global value for measuring inconsistency, whether cardinal or ordinal, is insufficient to locate all the inconsistent judgments present in a PCM. Hence, it is proposed to measure inconsistency at a fine-grained level to quantify the contribution of each judgment towards the overall inconsistency. A graph-theoretic approach is suggested for measuring level of inconsistency in the individual judgments. First, a generalized model is proposed to measure individual inconsistencies; and then, two new measures have been introduced: congruence for CC and dissonance for OC. The proposed measures are also applicable to incomplete sets of PC judgments without modification, unlike CR which requires a complete set of $\mathrm{PC}$ judgments. In addition, the congruence measure is applicable to any scale in a straight-forward manner, unlike CR which is only suitable for the linear and geometric scales.

The proposed measures are illustrated through examples, and it is concluded that the two matrices showing individual congruence and dissonance can be used to locate and (possibly) correct inconsistent (and/or outlying) judgments. The given examples have suggested measuring consistency as a pair of values i.e. $(\Theta, \Psi)$. It is suggested that the use of these matrices should form a useful addition to PC-based decision support tools. Further investigation is required to explore the properties of these two consistency measures. For example, the threshold of acceptance/rejection for $(\Theta, \Psi)$ should be investigated for different scales. A numerical comparison with other measures should be performed in order to compare these thresholds. 


\section{References}

Aguaron, J. and Moreno-Jimenez, J. M. (2003). The geometric consistency index: Approximated thresholds. European Journal of Operational Research, 147(1):137-145.

Ali, I., Cook, W. D., and Kress, M. (1986). On the Minimum Violations Ranking of a Tournament. Management Science, $32(6): 660-672$.

Belton, V. and Gear, T. (1983). On a short-coming of Saaty's method of analytic hierarchies. Omega, 11(3):228-230.

Bozóki, S., Fülöp, J., and Poesz, A. (2013). On reducing inconsistency of pairwise comparison matrices below an acceptance threshold.

Bozóki, S. and Rapcsak, T. (2008). On Saaty's and Koczkodaj's inconsistencies of pairwise comparison matrices. Journal of Global Optimization, 42(2):157-175.

Cao, D., Leung, L. C., and Law, J. S. (2008). Modifying inconsistent comparison matrix in analytic hierarchy process: A heuristic approach. Decision Support Systems, 44(4):944-953.

Choo, E. U. and Wedley, W. C. (2004). A common framework for deriving preference values from pairwise comparison matrices. Computers $\&$ Operations Research, 31(6):893-908.

Crawford, G. (1987). The geometric mean procedure for estimating the scale of a judgement matrix. Mathematical Modelling, $9(3-5): 327-334$.

Dyer, J. S. (1990). Remarks on the Analytic Hierarchy Process. Management Science, 36(3):249-258.

Ergu, D., Kou, G., Peng, Y., and Shi, Y. (2011). A simple method to improve the consistency ratio of the pair-wise comparison matrix in ANP. European Journal of Operational Research, 213(1):246-259.

Fedrizzi, M. (2012). Distance-based Characterization of Inconsistency in Pairwise Comparisons. Advances in Computational Intelligence: Communications in Computer and Information Science, 300:30-36.

Fedrizzi, M. and Giove, S. (2007). Incomplete pairwise comparison and consistency optimization. European Journal of Operational Research, 183(1):303-313.

Figueira, J., Greco, S., and Ehrgott, M. (2004). Multiple Criteria Decision Analysis: State of the Art Surveys. Springer.

Finan, J. S. and Hurley, W. J. (1999). Transitive calibration of the ahp verbal scale. European Journal of Operational Research, $112(2): 367-372$.

Gass, S. I. (1998). Tournaments, Transitivity and Pairwise Comparison Matrices. The Journal of the Operational Research Society, 49(6):616-624

Greco, S., Matarazzo, B., and Slowinski, R. (2010). Dominance-based rough set approach to preference learning from pairwise comparisons in case of decision under uncertainty. In Hallermeier, E., Kruse, R., and Hoffmann, F., editors, Computational Intelligence for Knowledge-Based Systems Design, volume 6178 of Lecture Notes in Computer Science, pages 584-594. Springer Berlin Heidelberg.

Harker, P. and Vargas, L. (1987). The theory of ratio scale estimation: Saaty's analytic hierarchy process. Management Science, 33(11):1383-1403

Harker, P. T. (1987). Incomplete pairwise comparisons in the analytic hierarchy process. Mathematical Modelling, 9(11):837848.

Hartvigsen, D. (2005). Representing the strengths and directions of pairwise comparisons. European Journal of Operational Research, 163(2):357-369. 
Herman, M. and Koczkodaj, W. (1996). A monte carlo study of pairwise comparison. Information Processing Letters, 57(1):2529.

Holsztynski, W. and Koczkodaj, W. W. (1996). Convergence of inconsistency algorithms for the pairwise comparisons. Information Processing Letters, 59(4):197-202.

Ishizaka, A., Balkenborg, D., and Kaplan, T. (2011). Influence of aggregation and measurement scale on ranking a compromise alternative in AHP. Journal of the Operational Research Society, 62:700-710.

Ishizaka, A. and Lusti, M. (2006). How to derive priorities in AHP: a comparative study. Central European Journal of Operations Research, 14(4):387-400.

Jensen, R. E. and Hicks, T. E. (1993). Ordinal data AHP analysis: A proposed coefficient of consistency and a nonparametric test. Mathematical and Computer Modelling, 17(4-5):135-150.

Kendall, M. G. (1955). Further Contributions to the Theory of Paired Comparisons. Biometrics, 11(1):43-62.

Kendall, M. G. and Smith, B. B. (1940). On the Method of Paired Comparisons. Biometrika, 31(3/4):324-345.

Koczkodaj, W. (1998). Testing the accuracy enhancement of pairwise comparisons by a monte carlo experiment. Journal of Statistical Planning and Inference, 69(1):21-31.

Koczkodaj, W. W. (1993). A new definition of consistency of pairwise comparisons. Mathematical and Computer Modelling, $18(7): 79-84$.

Koczkodaj, W. W. and Szarek, S. J. (2010). On distance-based inconsistency reduction algorithms for pairwise comparisons. Logic Journal of the IGPL, page jzp062.

Krantz, D. H. (1972). Measurement structures and psychological laws. Science, 175(4029):1427-1435.

Lipovetsky, S. and Conklin, W. M. (2002). Robust estimation of priorities in the AHP. European Journal of Operational Research, 137(1):110-122.

Lootsma, F. A. (1993). Scale sensitivity in the multiplicative ahp and smart. Journal of Multi-Criteria Decision Analysis, $2(2): 87-110$.

Monsuur, H. (1997). An intrinsic consistency threshold for reciprocal matrices. European Journal of Operational Research, $96(2): 387-391$.

Murphy, C. K. (1993). Limits on the analytic hierarchy process from its consistency index. European Journal of Operational Research, 65(1):138-139.

Saaty, T. (1980). The Analytic Hierarchy Process, Planning, Piority Setting, Resource Allocation. McGraw-Hill, New York. Saaty, T. (1994). Fundamentals of decision making and priority theory with the AHP. RWS Publications, Pittsburgh, PA.

Saaty, T. L. (1977). A scaling method for priorities in hierarchical structures. Journal of Mathematical Psychology, 15(3):234 -281 .

Saaty, T. L. (2008). Relative Measurement and Its Generalization in Decision Making. Why Pairwise Comparisons are Central in Mathematics for the Measurement of Intangible Factors - The Analytic $\{$ Hierarchy/Network $\}$ Process. RACSAM - Revista de la Real Academia de Ciencias Exactas, Fisicas y Naturales. Serie A. Matematicas, 102(2):251-318.

Salo, A. A. and Hamalainen, R. P. (1997). On the measurement of preferences in the analytic hierarchy process. Journal of $\{$ Multi-Criteria\} Decision Analysis, 6(6):309-319.

Seaman, M. A., Levin, J. R., and Serlin, R. C. (1991). New Developments in Pairwise Multiple Comparisons: Some Powerful and Practicable Procedures, volume 110. Psychological Bulletin.

Siraj, S., Mikhailov, L., and Keane, J. A. (2012). A heuristic method to rectify intransitive judgments in pairwise comparison 
matrices. European Journal of Operational Research, 216(2):420-428.

Siskos, Y., Grigoroudis, E., and Matsatsinis, N. (2005). Uta methods. In Multiple Criteria Decision Analysis: State of the Art Surveys, volume 78 of International Series in Operations Research 8 Management Science, pages 297-334. Springer New York.

Stein, W. E. and Mizzi, P. J. (2007). The harmonic consistency index for the analytic hierarchy process. European Journal of Operational Research, 177(1):488-497.

Stevens, S. (1961). Toward a resolution of the fechner-thurstone legacy. Psychometrika, 26(1):35-47.

Sugden, R. (1985). Why be Consistent? A Critical Analysis of Consistency Requirements in Choice Theory. Economica, $52(206): 167-183$.

Thurstone, L. L. (1927). A Law of Comparative Judgment. Psychological review, 34(4):273.

Vaidya, O. and Agarwal, S. (2006). Analytic hierarchy process: An overview of applications. European Journal of Operational Research, 169(1):1-29.

Vargas, L. (1990). An overview of the analytic hierarchy process and its applications. European Journal of Operational Research, 48(1).

Zeshui, X. and Cuiping, W. (1999). A consistency improving method in the analytic hierarchy process. European Journal of Operational Research, 116(2):443-449. 